\title{
Silver diamine fluoride, atraumatic restorations, and oral health-related quality of life - Results from the CariedAway cluster randomized trial
}

\author{
Ryan Richard Ruff*,a, Tamarinda J. Barry Godín ${ }^{\mathrm{a}}$, Topaz Murray Small ${ }^{\mathrm{a}}$, \\ Richard Niederman ${ }^{\mathrm{a}}$ \\ ${ }^{a}$ New York University College of Dentistry, Department of Epidemiology
}

\begin{abstract}
Objective: Silver diamine fluoride (SDF) is a non-surgical treatment for the arrest and prevention of dental caries that results in irreversible black staining of dental decay. The objective of this study was to evaluate the impact of SDF treatment on oral health-related quality of life (OHRQoL) relative to a standard package of glass ionomer sealants and atraumatic restorative treatment. Methods: CariedAway is a pragmatic, longitudinal, cluster-randomized noninferiority trial of non-surgical interventions for caries. Secondary study outcomes included OHRQoL and academic performance. Oral health-related quality of life was measured at each study visit using the Child Oral Health Impact Profile. Change in OHRQoL was assessed using linear regression and non-inferiority was determined using t-tests. Results: Untreated decay at baseline was associated with signifcantly worse ORHQoL and treatment in both groups resulted in incremental improvement. Quality of life in children receiving silver diamine fluoride was non-inferior to those receiving sealants and ART at least six months post-treatment. Additionally, change in OHRQoL did not depend on the severity of baseline decay. Conclusions: ORHQOL is related to untreated dental caries, however no appreciable change was observed following SDF treatment relative to standard preventive therapies.
\end{abstract}

\section{Introduction}

Dental caries is the most prevalent childhood disease in the world (Bernabe et al., 2020), found across all age groups and most prominent among lowincome populations (Frencken et al., 2017). Untreated caries has been shown to develop into pain and systemic infection, potentially resulting in functional and/or psychosocial impairment (Mathur and Dhillon, 2018). Much of the

\footnotetext{
${ }^{*}$ Corresponding Author

Email addresses: ryan.ruff@nyu.edu (Ryan Richard Ruff), t.barrygodin@nyu.edu (Tamarinda J. Barry Godín), topaz.murray@nyu.edu (Topaz Murray Small), r.niederman@nyu.edu (Richard Niederman)
}

Preprint submitted to medRxiv

April 14, 2021 
disproportionate burden of disease amongst vulnerable groups, such as lowincome and minority populations, is due to lower accessibility and utilization of traditional dental services (Dye et al., 2012; Griffin et al., 2016; Treadwell, 2017). As a result, the use of non-surgical treatments such as silver diamine fluoride (SDF) is increasing. Silver diamine fluoride is a noninvasive method to prevent and arrest caries that can be efficiently applied in community settings (Contreras et al., 2017; Oliveira et al., 2019; Wierichs and Meyer-Lueckel, 2015), but results in permanent black staining of dental decay.

Oral health-related quality of life (OHRQoL) is a multidimensional construct consisting of subjective evaluations of oral health, functional well-being, emotional well-being, satisfaction with care, and sense of self (Ruff et al., 2017). Caries may have a negative impact in oral health-related quality of life in preschool children (Nora et al., 2018), children aged 3-12 years and adults (Aimee et al., 2017; Haag et al., 2017; Moghaddam et al., 2020). High caries experience (Chaffee et al., 2017) and untreated caries (Fernandes et al., 2017) both exhibit reduced OHRQoL, regardless of measurement used (Arrow, 2017; García-Pérez et al., 2017). Despite a high oral disease burden (Ahluwalia and Sadowsky, 2003), research on quality of life and caries in black and Hispanic/Latino populations is limited (Broder et al., 2000; Southward et al., 2008); evidence on silver diamine fluoride and quality of life presents conflicting results with treatment shown to either improve or have no effect on OHRQoL in children (Duangthip et al., 2019; Jiang et al., 2020, 2019; Rodrigues et al., 2020; Sihra et al., 2020; Vollu et al., 2019); and the impact on OHRQoL comparing SDF to atraumatic restorative treatment is unclear (Rodrigues et al., 2020; Vollu et al., 2019).

CariedAway is an active randomized controlled trial of non-surgical interventions for the prevention and treatment of caries in children aged 5-13 years (Ruff and Niederman, 2018), specifically silver diamine fluoride, sealants, and atraumatic restorative treatment. The CariedAway study also aims to evaluate the effects of treatment on quality of life, academic performance, and school attendance. The objectives of this paper are to assess (1) the associations between oral health-related quality of life and dental caries and (2) the short term effects of non-surgical treatment for caries on oral health-related quality of life.

\section{Methods}

Ethical approval for the CariedAway clinical trial was obtained from the New York University School of Medicine Institutional Review Board (i17-00578). A previously published trial protocol contains additional study-related information (Ruff and Niederman, 2018) and the trial is registered at www.clinicaltrials.gov (\#NCT03442309). Preliminary clinical results are forthcoming.

\subsection{Design}

CariedAway is a longitudinal, cluster-randomized, single-blind, pragmatic trial with the primary objective of evaluating the non-inferiority of non-surgical treatments for dental caries. Any school in New York City with a student 
population of at least $80 \%$ receiving free or reduced lunch and at least $50 \%$ Hispanic/Latino or black was eligible to participate in the study. All children in enrolled schools were provided informed consent, there were no inclusion criteria for child-level enrollment. Any subject with parental informed consent and child assent was randomly assigned to treatment and received care. Treatment was provided in scheduled six-month intervals.

\subsection{Interventions}

Interventions included two separate packages of non-therapeutic treatments for dental caries: a simple combination of fluoride varnish applied to all teeth and silver diamine fluoride applied to all pits and fissures and asymptomatic cavitated lesions of bicuspids and molars, and a complex combination consisting of the same application of fluoride varnish, glass ionomer sealants applied to pits and fissures of bicuspids and molars, and use of atraumatic restorative treatment on all frank asymptomatic cavitated lesions. Caries diagnosis followed the International Caries Detection and Assessment System (ICDAS) adapted criteria and the diagnostic and treatment protocol is previously described (Ruff and Niederman, 2018).

\subsection{Randomization}

Enrolled schools were block randomized in a 1:1 allocation ratio.

\subsection{Data Collection}

At each observation, study clinicians performed a full visual-tactile oral examination and recorded the missing, sound, decayed, or filled status of every tooth surface. Clinicians were standardized and calibrated prior to observing subjects. Following the oral examination and application of treatments, children were asked to participate in answering the COHIP-SF quality of life survey. The clinician would then read each question aloud to subjects who would then note their answers on a provided tablet computer.

\subsection{Outcomes}

Oral health-related quality of life was assessed using the Child Oral Health Impact Profile Short Form (COHIP-SF), consisting of 19 questions assessing oral health, functional well-being, socio-emotional well-being, school-environment, and self-image (Broder et al., 2012; Broder and Wilson-Genderson, 2007). Examiners asked participants each COHIP-SF question (e.g., "Have you in the past three months had pain in your teeth or a toothache"), and children responded by touching an indicator on the tablet or communicating their answer ("Never," "Almost never," "Sometimes," "Fairly often," "Almost all of the time") to the examiner. A global quality of life indicator measuring perceived change from the previous observation was also used. For the global QoL question, participants were asked "On a scale of 0 to 100 where 0 is worst and 100 is best, how would you rate your quality of life?" Subjects were then shown a visual scale on a horizontal axis ranging from 0 to 10 in 10-point intervals, with "worst" at the zero 
point, "medium" at the 50th point, and "best" at the 100th point. Participants then tapped their answer at the point of the scale directly on the tablet. Not all children received the COHIP instrument: fifty percent of participants in the CariedAway trial were randomly assigned within each group at baseline to receive the quality of life assessment. Assigned children then received the same QoL instrument at every successive treatment.

\subsection{Covariates}

Demographic information including age, sex, and race/ethnicity were obtained from informed consent documents or school records. A unique identification number maintained by the Office of School Health at the New York City Department Health and Mental Hygiene and New York City Department of Education was similarly used as the patient record number for this study.

\subsection{Statistical Analysis}

Analysis was restricted to only those subjects between the ages of 5 and 13 years at time of observation. Subjects were analyzed using intent to treat: any child who may have switched schools that was randomized to a different treatment arm was analyzed according to his or her original assignment. Baseline descriptive statistics for sociodemographic variables and COHIP scores were computed. The association between dental caries at baseline and initial quality of life was assessed using linear regression. Following baseline analyses, subjects were ordered sequentially by visit date and any child without two completed visits was removed from the analytic sample. Within-group differences for treatment groups used paired samples t-tests. Post-treatment analysis of the caries-QoL association was assessed using linear regression, adjusting for treatment group, baseline COHIP scores, and demographic variables. The intraclass correlation coefficient for subjects nested within schools was estimated using an interceptonly mixed effects multilevel model. All analyses adjusted for the clustering effect of schools. The non-inferiority of SDF therapy compared to sealants/ART on quality of life was determined by calculating the confidence interval for the difference in mean COHIP scores across treatment group. As per the original study protocol, a non-inferiority margin of ten was used.

\section{Results}

1323 subjects completed the quality of life assessment at baseline, 160 of which $(12 \%)$ completed a second-follow up at least six months following treatment with either silver diamine fluoride or sealants/ART, prior to study suspension due to the impact of the SARS-CoV-2 pandemic. The sample was approximately $53 \%$ female and consisted of $39 \%$ Hispanic/Latino, $15 \%$ black, $4 \%$ white, and $1.4 \%$ Asian (Table 1). The overall prevalence of any untreated decay on any tooth (deciduous or permanent) was $25.8 \%$ with an average per-person number of decayed teeth of $0.45(\mathrm{SD}=1.1)$. The average time between treatments was 189 days. The intraclass correlation was $3.26 \%$. 
Table 1: Baseline sample demographics and clinical outcomes $(\mathrm{N}=1323)$

\begin{tabular}{|c|c|c|}
\hline Variable & N/Mean & $\% / \mathrm{SD}$ \\
\hline \multicolumn{3}{|l|}{ Sex } \\
\hline Female & 700 & 52.91 \\
\hline \multicolumn{3}{|l|}{ Etnicity } \\
\hline Hispanic & 513 & 38.78 \\
\hline Non-Hispanic & 105 & 7.94 \\
\hline $\mathrm{N} / \mathrm{A}$ & 705 & 53.29 \\
\hline \multicolumn{3}{|l|}{ Race } \\
\hline Black & 203 & 15.34 \\
\hline Asian & 19 & 1.44 \\
\hline Multirace & 34 & 2.57 \\
\hline White & 56 & 4.23 \\
\hline $\mathrm{N} / \mathrm{A}$ & 984 & 74.38 \\
\hline \multicolumn{3}{|l|}{ Clinical Indicators } \\
\hline Untreated decay & 341 & 25.77 \\
\hline \# Decayed teeth & 0.49 & $1.09(\mathrm{SD})$ \\
\hline
\end{tabular}

Table 2: Baseline decay and OHRQoL

\begin{tabular}{ccc}
\hline Model & B & $95 \%$ CI \\
\hline Any Decay & 3.76 & $2.37,5.14$ \\
Total Decay & 1.37 & $0.82,1.92$ \\
\hline
\end{tabular}

Children with untreated decay at baseline, irrespective of treatment assignment, scored significantly worse on oral health-related quality of life $(\mathrm{B}=3.76$, $95 \% \mathrm{CI}=2.37,5.14)$. Adjusting for differences in race and ethnicity, each decayed tooth was associated with 1.37 point increase in COHIP scores, where higher scores indicate worse OHRQoL (Table 2). There were no baseline differences in OHRQoL by treatment group ( $\mathrm{B}=-0.61,95 \% \mathrm{CI}=-4.17,2.94)$. Across both SDF and sealant/ART groups, average OHRQoL improved following treatment: COHIP scores improved from an average of $16.28(\mathrm{SD}=11.32)$ in children receiving silver diamine fluoride at baseline to $14.51(\mathrm{SD}=11.83)$ at follow-up, while those receiving sealants and atraumatic restorative treatments improved from $16.90(\mathrm{SD}=10.26)$ to $16.38(\mathrm{SD}=11.33)$. Following at least six months post-treatment, there were no differences in OHRQoL by treatment group (Table 3), adjusting for baseline COHIP scores, the number of decayed teeth, and sociodemographic factors $(\mathrm{B}=-.083,95 \% \mathrm{CI}=-3.89,2.23)$.

Comparisons of the analytic sample at the follow-up visit indicate that selfreported oral health-related quality of life in children receiving silver diamine fluoride is non-inferior to those receiving traditional glass ionomer sealants and 
Table 3: SDF vs ITR for OHRQoL (analytic sample)

\begin{tabular}{ccc}
\hline Variable & $\mathrm{B}$ & $95 \%$ CI \\
\hline SDF & -0.83 & $-3.90,2.23$ \\
Baseline OHRQoL & 0.68 & $0.55,0.81$ \\
Total decay & 0.34 & $-1.91,2.60$ \\
\hline a
\end{tabular}

${ }^{\mathrm{a}}$ Models also adjusted for race and age

ART (mean difference $=1.87,95 \% \mathrm{CI}=-1.92,5.66$ ). The point estimate favors silver diamine fluoride however the confidence interval is below the non-inferiority margin. Additionally, there was no significant interaction: no treatment differences were found amongst only subjects with baseline caries.

\section{Discussion}

As arresting and preventive agents for dental caries, atraumatic restorative treatments and glass ionomer sealants are visually imperceptible when compared to traditional composite restorations. In contrast, application of silver diamine fluoride results in permanent black staining of dental decay and superficial staining of the oral mucosa. Notably, perceptions of self are affected by facial aesthetics, being previously observed in adolescents seeking orthodontic treatment and in children with preexisting orofacial anomalies (Phillips and Beal, 2009; Ruff et al., 2016a). As approximately 25\% of CariedAway participants had untreated decay at baseline with significantly lower OHRQoL than caries-free children, concerns regarding the aesthetic impact of SDF, despite demonstrated clinical and economic benefits (Contreras et al., 2017; Yeung and Argáez, 2017), may be justified.

Oral health-related quality of life slightly improved following treatment with either silver diamine fluoride or sealants/ART, and children receiving SDF exceeded the minimally important difference threshold of the COHIP-SF necessary for patient-centered clinically meaningful change (Ruff et al., 2016b). Results further suggest that OHRQoL in children receiving SDF was non-inferior to those of children receiving ART/sealants. These results are consistent with other studies of SDF in children reporting similar effects when compared to alternative treatments, such as ART, fluoride varnish, or placebo (Duangthip et al., 2019; Jiang et al., 2020, 2019; Sihra et al., 2020; Vollu et al., 2019).

Clinical application of silver diamine fluoride in the CariedAway trial does not include anterior teeth; SDF is often applied to posterior teeth in order to avoid impacts on facial aesthetics. In children aged 5-9 years, the global prevalence rate of caries in deciduous teeth exceeds $40 \%$ (Bernabe et al., 2020). However, decay most often occurs in the occlusal surface of molars and pre-molars (Demirci et al., 2010), thus a restriction to posterior application will still treat a majority of underlying disease. In previous studies of SDF, caregivers of children with untreated caries were more accepting of the staining effect when applied to 
posterior lesions, if the child had a history of behavioral issues when treated by a dentist, or if more invasive measures, such as anesthesia, would be required (Seifo et al., 2020). Anterior application of silver diamine fluoride may be acceptable for deciduous teeth due to expected exfoliation, but more aesthetically pleasing alternatives may be required for permanent anterior teeth in adolescents.

The focus of this analysis was on the potential short-term impact of SDF application on oral health-related quality of life, relative to more traditional nontherapeutic caries treatments. It may be the case that the staining effect of SDF, even when confined to posterior teeth, becomes more appreciated with longer rates of follow-up or when children progress into adolescence where facial aesthetics may be of greater concern. Subsequent objectives of the CariedAway trial are to assess longitudinal change in OHRQoL and identify latent trajectories of quality of life. Additionally, as overall oral health-related quality of life has been shown to be responsive to the severity of dental caries (Corrêa-Faria et al., 2018), the long-term impact on OHRQoL following treatment with SDF may behave in a similar manner.

Silver diamine fluoride can be applied in significantly less time than atraumatic restorative treatments (Vollu et al., 2019) and does not require the same degree of clinical training, suggesting that SDF is more efficient as a pragmatic treatment for caries. For example, some states authorize registered nurses to provide SDF under the supervision of a licensed general dentist. Additionally, the non-invasive nature of SDF as an arresting agent, combined with its secondary preventive effects, make it an attractive alternative to more traditional nonsurgical interventions (Horst and Heima, 2019). Our results suggest that children do not perceive any negative impacts on oral health-related quality of life approximately six months following application. These findings, combined with documented evidence of safety and clinical efficacy, further support the continued use of silver diamine fluoride.

\section{Supporting Information}

\subsection{Funding}

This work was supported in part by an award from the Patient Centered Outcomes Research Institute (\#PCS160936724). The content is solely the responsibility of the authors and does not necessarily reflect the official views of the funding organization, New York University, or the NYU College of Dentistry.

\subsection{Conflict of Interest}

The authors report no conflicts of interest.

\subsection{Acknowledgements}

The authors would like to acknowledge the following members of the CariedAway project team: Rachel Whittemore, Nydia Santiago-Galvin, Haley Gibbs, Catherine McGowan, Priyanka Sharma, and those providing clinical care. 


\section{References}

Ahluwalia, K.P., Sadowsky, D., 2003. Oral disease burden and dental services utilization by latino and african-american seniors in northern manhattan. J Community Health 28, 267-80. doi:10.1023/a:1023938108988

Aimee, N.R., Wijk, A.J. van, Maltz, M., Varjao, M.M., Mestrinho, H.D., Carvalho, J.C., 2017. Dental caries, fluorosis, oral health determinants, and quality of life in adolescents. Clin Oral Investig 21, 1811-1820. doi:10.1007/s00784016-1964-3

Arrow, P., 2017. Dental enamel defects, caries experience and oral health-related quality of life: A cohort study. Aust Dent J 62, 165-172. doi:10.1111/adj.12449

Bernabe, E., Marcenes, W., Hernandez, C.R., Bailey, J., Abreu, L.G., Alipour, V., Amini, S., Arabloo, J., Arefi, Z., Arora, A., Ayanore, M.A., Bärnighausen, T.W., Bijani, A., Cho, D.Y., Chu, D.T., Crowe, C.S., Demoz, G.T., Demsie, D.G., Dibaji Forooshani, Z.S., Du, M., El Tantawi, M., Fischer, F., Folayan, M.O., Futran, N.D., Geramo, Y.C.D., Haj-Mirzaian, A., Hariyani, N., Hasanzadeh, A., Hassanipour, S., Hay, S.I., Hole, M.K., Hostiuc, S., Ilic, M.D., James, S.L., Kalhor, R., Kemmer, L., Keramati, M., Khader, Y.S., Kisa, S., Kisa, A., Koyanagi, A., Lalloo, R., Le Nguyen, Q., London, S.D., Manohar, N.D., Massenburg, B.B., Mathur, M.R., Meles, H.G., Mestrovic, T., Mohammadian-Hafshejani, A., Mohammadpourhodki, R., Mokdad, A.H., Morrison, S.D., Nazari, J., Nguyen, T.H., Nguyen, C.T., Nixon, M.R., Olagunju, T.O., Pakshir, K., Pathak, M., Rabiee, N., Rafiei, A., Ramezanzadeh, K., Rios-Blancas, M.J., Roro, E.M., Sabour, S., Samy, A.M., Sawhney, M., Schwendicke, F., Shaahmadi, F., Shaikh, M.A., Stein, C., Tovani-Palone, M.R., Tran, B.X., Unnikrishnan, B., Vu, G.T., Vukovic, A., Warouw, T.S.S., Zaidi, Z., Zhang, Z.J., Kassebaum, N.J., 2020. Global, regional, and national levels and trends in burden of oral conditions from 1990 to 2017: A systematic analysis for the global burden of disease 2017 study. J Dent Res 99, 362-373. doi:10.1177/0022034520908533

Broder, H.L., Slade, G., Caine, R., Reisine, S., 2000. Perceived impact of oral health conditions among minority adolescents. J Public Health Dent 60, 189-92. doi:10.1111/j.1752-7325.2000.tb03326.x

Broder, H.L., Wilson-Genderson, M., 2007. Reliability and convergent and discriminant validity of the child oral health impact profile (COHIP child's version). Community Dent Oral Epidemiol 35 Suppl 1, 20-31. doi:10.1111/j.16000528.2007.0002.x

Broder, H.L., Wilson-Genderson, M., Sischo, L., 2012. Reliability and validity testing for the child oral health impact profile-reduced (COHIP-SF 19). J Public Health Dent 72, 302-12. doi:10.1111/j.1752-7325.2012.00338.x

Chaffee, B.W., Rodrigues, P.H., Kramer, P.F., Vitolo, M.R., Feldens, C.A., 2017. Oral health-related quality-of-life scores differ by socioeconomic status and caries experience. Community Dent Oral Epidemiol 45, 216-224. doi:10.1111/cdoe.12279

Contreras, V., Toro, M.J., Elías-Boneta, A.R., Encarnación-Burgos, A., 2017. Effectiveness of silver diamine fluoride in caries prevention and arrest: A 
systematic literature review. Gen Dent 65, 22-29.

Corrêa-Faria, P., Daher, A., Freire, M., Abreu, M. de, Bönecker, M., Costa, L.R., 2018. Impact of untreated dental caries severity on the quality of life of preschool children and their families: A cross-sectional study. Qual Life Res 27, 3191-3198. doi:10.1007/s11136-018-1966-5

Demirci, M., Tuncer, S., Yuceokur, A.A., 2010. Prevalence of caries on individual tooth surfaces and its distribution by age and gender in university clinic patients. Eur J Dent 4, 270-9.

Duangthip, D., Gao, S.S., Chen, K.J., Lo, E.C.M., Chu, C.H., 2019. Oral healthrelated quality of life of preschool children receiving silver diamine fluoride therapy: A prospective 6-month study. J Dent 81, 27-32. doi:10.1016/j.jdent.2018.12.004

Dye, B.A., Li, X., Thorton-Evans, G., 2012. Oral health disparities as determined by selected healthy people 2020 oral health objectives for the united states, 2009-2010. NCHS Data Brief 1-8.

Fernandes, I.B., Pereira, T.S., Souza, D.S., Ramos-Jorge, J., Marques, L.S., Ramos-Jorge, M.L., 2017. Severity of dental caries and quality of life for toddlers and their families. Pediatr Dent 39, 118-123.

Frencken, J.E., Sharma, P., Stenhouse, L., Green, D., Laverty, D., Dietrich, T., 2017. Global epidemiology of dental caries and severe periodontitis - a comprehensive review. J Clin Periodontol 44 Suppl 18, S94-S105. doi:10.1111/jcpe.12677

García-Pérez, Á., Irigoyen-Camacho, M.E., Borges-Yáñez, S.A., Zepeda-Zepeda, M.A., Bolona-Gallardo, I., Maupomé, G., 2017. Impact of caries and dental fluorosis on oral health-related quality of life: A cross-sectional study in schoolchildren receiving water naturally fluoridated at above-optimal levels. Clin Oral Investig 21, 2771-2780. doi:10.1007/s00784-017-2079-1

Griffin, S.O., Wei, L., Gooch, B.F., Weno, K., Espinoza, L., 2016. Vital signs: Dental sealant use and untreated tooth decay among u.s. School-aged children. MMWR Morb Mortal Wkly Rep 65, 1141-1145. doi:10.15585/mmwr.mm6541e1

Haag, D.G., Peres, K.G., Balasubramanian, M., Brennan, D.S., 2017. Oral conditions and health-related quality of life: A systematic review. J Dent Res 96, 864-874. doi:10.1177/0022034517709737

Horst, J.A., Heima, M., 2019. Prevention of dental caries by silver diamine fluoride. Compend Contin Educ Dent 40, 158-163; quiz 164.

Jiang, M., Wong, M.C.M., Chu, C.H., Dai, L., Lo, E.C.M., 2019. Effects of restoring SDF-treated and untreated dentine caries lesions on parental satisfaction and oral health related quality of life of preschool children. J Dent 88, 103171. doi:10.1016/j.jdent.2019.07.009

Jiang, M., Xie, Q.Y., Wong, M.C.M., Chu, C.H., Lo, E.C.M., 2020. Association between dental conditions, sliver diamine fluoride application, parental satisfaction, and oral health-related quality of life of preschool children. Clin Oral Investig. doi:10.1007/s00784-020-03542-8

Mathur, V.P., Dhillon, J.K., 2018. Dental caries: A disease which needs attention. Indian J Pediatr 85, 202-206. doi:10.1007/s12098-017-2381-6

Moghaddam, L.F., Vettore, M.V., Bayani, A., Bayat, A.H., Ahounbar, E., Hemmat, M., Armoon, B., Fakhri, Y., 2020. The association of oral health 
status, demographic characteristics and socioeconomic determinants with oral health-related quality of life among children: A systematic review and meta-analysis. BMC Pediatr 20, 489. doi:10.1186/s12887-020-02371-8

Nora, A.D., Silva Rodrigues, C. da, Oliveira Rocha, R. de, Soares, F.Z.M., Minatel Braga, M., Lenzi, T.L., 2018. Is caries associated with negative impact on oral health-related quality of life of pre-school children? A systematic review and meta-analysis. Pediatr Dent 40, 403-411.

Oliveira, B.H., Rajendra, A., Veitz-Keenan, A., Niederman, R., 2019. The effect of silver diamine fluoride in preventing caries in the primary dentition: A systematic review and meta-analysis. Caries Res 53, 24-32. doi: $10.1159 / 000488686$

Phillips, C., Beal, K.N., 2009. Self-concept and the perception of facial appearance in children and adolescents seeking orthodontic treatment. Angle Orthod 79, 12-6. doi:10.2319/071307-328.1

Rodrigues, G.F., Costa, T.D.C., Massa, G.D.S., Vollu, A.L., Barja-Fidalgo, F., Fonseca-Goncalves, A., 2020. Oral health-related quality of life in preschool children after silver diamine fluoride versus atraumatic restorative treatments. Pediatr Dent 42, 373-379.

Ruff, R.R., Niederman, R., 2018. Silver diamine fluoride versus therapeutic sealants for the arrest and prevention of dental caries in low-income minority children: Study protocol for a cluster randomized controlled trial. Trials 19, 523. doi:10.1186/s13063-018-2891-1

Ruff, R.R., Sischo, L., Broder, H., 2016a. Resiliency and socioemotional functioning in youth receiving surgery for orofacial anomalies. Community Dent Oral Epidemiol 44, 371-80. doi:10.1111/cdoe.12222

Ruff, R.R., Sischo, L., Broder, H.L., 2016b. Minimally important difference of the Child Oral Health Impact Profile for children with orofacial anomalies. Health Qual Life Outcomes 14, 140.

Ruff, R.R., Sischo, L., Chinn, C.H., Broder, H.L., 2017. Development and validation of the child oral health impact profile - preschool version. Community Dent Health 34, 176-182. doi:10.1922/CDH_4110Ruff07

Seifo, N., Robertson, M., MacLean, J., Blain, K., Grosse, S., Milne, R., Seeballuck, C., Innes, N., 2020. The use of silver diamine fluoride (SDF) in dental practice. Br Dent J 228, 75-81. doi:10.1038/s41415-020-1203-9

Sihra, R., Schroth, R.J., Bertone, M., Martin, H., Patterson, B., Mittermuller, B.A., Lee, V., Patterson, B., Moffatt, M.E., Klus, B., Fontana, M., Robertson, L., 2020. The effectiveness of silver diamine fluoride and fluoride varnish in arresting caries in young children and associated oral health-related quality of life. J Can Dent Assoc 86, k9.

Southward, L.H., Robertson, A., Edelstein, B.L., Hanna, H., Wells-Parker, E., Baggett, D.H., Eklund, N.P., Crall, J.J., Silberman, S.L., Parrish, D.R., 2008. Oral health of young children in mississippi delta child care centers: A second look at early childhood caries risk assessment. J Public Health Dent 68, 188-95. doi:10.1111/j.1752-7325.2007.00061.x

Treadwell, H.M., 2017. The nation's oral health inequities: Who cares. Am J Public Health 107, S5. doi:10.2105/AJPH.2017.303957 
medRxiv preprint doi: https://doi.org/10.1101/2021.04.19.21255478; this version posted April 20, 2021. The copyright holder for this preprint (which was not certified by peer review) is the author/funder, who has granted medRxiv a license to display the preprint in perpetuity.

It is made available under a CC-BY-NC-ND 4.0 International license .

Vollu, A.L., Rodrigues, G.F., Rougemount Teixeira, R.V., Cruz, L.R., Dos Santos Massa, G., Lima Moreira, J.P. de, Luiz, R.R., Barja-Fidalgo, F., FonsecaGoncalves, A., 2019. Efficacy of 30. J Dent 88, 103165. doi:10.1016/j.jdent.2019.07.003

Wierichs, R.J., Meyer-Lueckel, H., 2015. Systematic review on noninvasive treatment of root caries lesions. J Dent Res 94, 261-71. doi:10.1177/0022034514557330

Yeung, S.S.T., Argáez, C., 2017. CADTH rapid response reports, in: Silver Diamine Fluoride for the Prevention and Arresting of Dental Caries or Hypersensitivity: A Review of Clinical Effectiveness, Cost-Effectiveness and Guidelines. Canadian Agency for Drugs; Technologies in Health Copyright ( 2017 Canadian Agency for Drugs; Technologies in Health., Ottawa (ON). 